\title{
On the Influence of Magnetic Field on Accretion Processes in CVs
}

\author{
D. V. Bisikalo${ }^{1}$, A. G. Zhilkin ${ }^{1}$ \\ ${ }^{1}$ Institute of Astronomy RAS, Moscow, Russia \\ Corresponding author: bisikalo@inasan.ru
}

\begin{abstract}
We consider the influence of such parameters as the value of the proper magnetic field $B_{a}$ and the spin-rotation velocity of the white dwarf on accretion processes in CVs. The results of 3D MHD simulations have shown that the accretion rate is a non-monotonic function of $B_{a}$ : with growing $B_{a}$ it raises in the intermediate polars and decreases in the polars. The maximal accretion rate occurs in the systems, transiting from the stage of intermediate polars to polars; it's value reaches $\sim 60 \%$ of the initially set mass transfer rate. We have also shown that the acretion rate decreases with the growing spin-rotation velocity of the white dwarf.
\end{abstract}

Keywords: cataclysmic variables - intermediate polars - polars - accretion - MHD simulations.

\section{Introduction}

Two main classes of CVs where magnetic field significantly influences accretion processes are the intermediate polars and polars [1]. These are semi-detached binary systems, consisting of a low-mass late type star (donor-star) and a white dwarf (accretor).

In the polars, white dwarfs possess strong magnetic fields ( $B_{a} \approx 10^{7}-10^{8} \mathrm{G}$ on the surface). Observations show that no accretion disks form in polars. Instead, the material, issuing from the $L_{1}$ point, forms a collimated stream, that flows onto one of the magnetic poles of the accretor along magnetic field lines[1, 2]. In the intermediate polars, white dwarfs possess relatively weak magnetic fields $\left(B_{a} \approx 10^{4}-10^{6} \mathrm{G}\right.$ on the surface). These systems occupy an intermediate stage between the polars and non-magnetic CVs. We should note that in these systems the spin-rotation periods of the primaries may be significantly shorter than the orbital periods [3].

In our previous works $[4,5]$ we, by means of $3 \mathrm{D}$ MHD simulations, studied the flow structure of the intermediate polars and polars, having various surface magnetic fields of the accretors $B_{a}$, ranging from $10^{5}$ to $10^{8} \mathrm{G}$. This allowed us to investigate conditions of accretion disk formation and to find a criterion, separating two types of the flow, corresponding to the intermediate polars and polars. Besides, in [5] we also investigated how the spin-rotation velocity of the accretor influences the MHD flow structure in the system.In this paper we summarize the results of our previous research and show how the accretion rate varies, depending on two parameters as the magnetic induction on the surface of the white dwarf and its spin-rotation velocity.

\section{What Happens with the Flow Structure if $B_{a}$ Grows}

To investigate how the value of the accretor's surface magnetic field influences MHD flow structure in a close binary system we have conducted 7 computational runs with various $B_{a}: 10^{5} \mathrm{G}$ (model 1 ); $5 \times 10^{5} \mathrm{G}$ (model 2 ); $10^{6} \mathrm{G}$ (model 3); $5 \times 10^{6} \mathrm{G}$ (model 4); $10^{7} \mathrm{G}$ (model 5); $5 \times 10^{7} \mathrm{G}$ (model 6 ); and $10^{8} \mathrm{G}$ (model 7). We suppose that the accretor's magnetic field is of dipole type and the axis of the field is inclined with respect to the accretor's rotation axis by $\theta=30^{\circ}$.

For the computations we used our method of investigations of mass transfer processes in semi-detached binary systems where the accretors possess strong magnetic fields $[4,5]$. The method is based on the idea that plasma dynamics is determined by the relatively slow average motion of plasma against a background of which very rapid MHD waves propagate. The equations, describing the slow motion of plasma, are derived via a procedure of averaging over the rapidly propagating pulsations. Strong external magnetic field in this approach plays a role of an effective liquid interacting with plasma. In the equation of motion the mean electromagnetic force is analogous to the friction force working in multi-component plasma. In our numerical model we take into account processes of the magnetic field diffusion caused by the currents' dissipation in turbulent vortices and magnetic buoyancy. Besides, when averaging the induction equation over the rapidly propagating MHD waves we introduce an additional source of magnetic field dissipation (wave dissipation).

In order to see how namely the magnetic induction influences the solution we fix all the other param- 
eters. The computations have been conducted for a close binary system, having parameters of the SS Cygni (see [6]). The donor-star (red dwarf) in this system has the mass $M_{d}=0.56 M_{\odot}$ and the effective temperature of $4000 \mathrm{~K}$. The accretor (white dwarf) has the mass $M_{a}=0.97 M_{\odot}$ and the temperature of $37000 \mathrm{~K}$. The orbital period of the system is $P_{\text {orb }}=6.6$ hours and its binary separation is $A=2.05 R_{\odot}$. We have supposed that the accretion disk forms in the system for the first time so the accretor's spin-rotation is synchronous, i.e. $P_{\text {spin }}=P_{\text {orb }}$. The influence of the accretor's spinrotation on a formed accretion disk is considered in the next section.

The obtained solutions can be divided into two groups. The first group contains models 1,2 , and 3 with relatively weak magnetic fields where accretion disks form. These models correspond to the intermediate polars. In the models of the second group (models 4-7) magnetic fields are strong and there are no accretion disks formed. These models represent the polars.

The typical flow structure in the intermediate polars is shown in Fig. 1, where we plotted an iso-surface of density $\operatorname{logarithm} \lg \rho=-4.5$ (in units of $\rho\left(L_{1}\right)$ ) obtained in the model $1\left(B_{a}=10^{5} \mathrm{G}\right)$. In Figure we also show the magnetic flow lines originating on the accretor's surface, accretor's rotation axis (thin straight line) and magnetic axis (bold inclined line). We note that all the gas dynamic structures found in our previous studies $[7,8,5]$, including the accretion disk and shock waves exist in the obtained solution. Near the accretor the magnetosphere forms and material is accreted via funnel flows.

With growing magnetic field the outer radius of the disk significantly decreases. The efficiency of the magnetic breaking and angular momentum transfer increases. The region of the magnetosphere becomes significantly larger. Finally, when $B_{a}=10^{6} \mathrm{G}$ (model 3, Fig. 2) the accretion disk almost degenerates. The material makes only 1-2 cycles before falling onto the accretor. To describe this flow structure a term "spiralodisk" is more appropriate, since the velocity distribution in this structure strongly differs from the Keplerian. The outer radius of this "spiralo-disk" is approximately $0.1 \mathrm{~A}$ and the inner radius is determined by the size of the magnetosphere. A significant part of the "spiralo-disk" is accretion funnel flows. We can state that this solution is an ultimate case of the intermediate polars.

To determine a criterion, separating the intermediate polars and polars let us consider behavior of the gas stream, issuing from the $L_{1}$ point into the Roche lobe of the accretor. Taking into account that the gas in the stream moves with supersonic velocity we can consider its behavior using the ballistic approach and omitting the effects of pressure and magnetic field $[9,10]$.

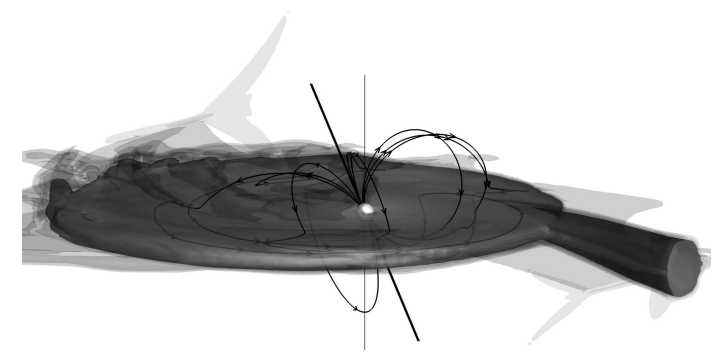

Figure 1: 3D flow structure for the model 1 with $B_{a}=10^{5} \mathrm{G}$. Iso-surface of density $(\lg \rho=-4.5$ in units of $\left.\rho\left(L_{1}\right)\right)$ and magnetic field lines are shown. The accretor's rotation axis is shown by the thin straight line, magnetic axis - by the bold inclined line.

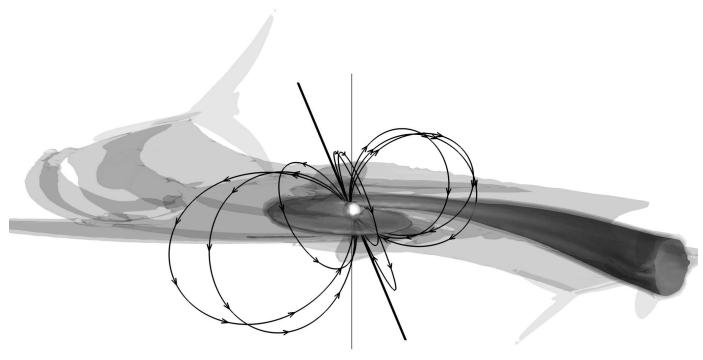

Figure 2: The same as in Fig. 1 for the model 3 with $B_{a}=10^{6} \mathrm{G}$.

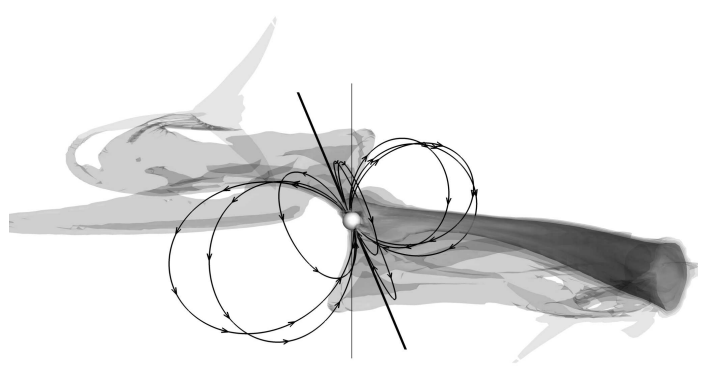

Figure 3: The same as in Fig. 1 for the model 5 with $B_{a}=10^{7} \mathrm{G}$. 

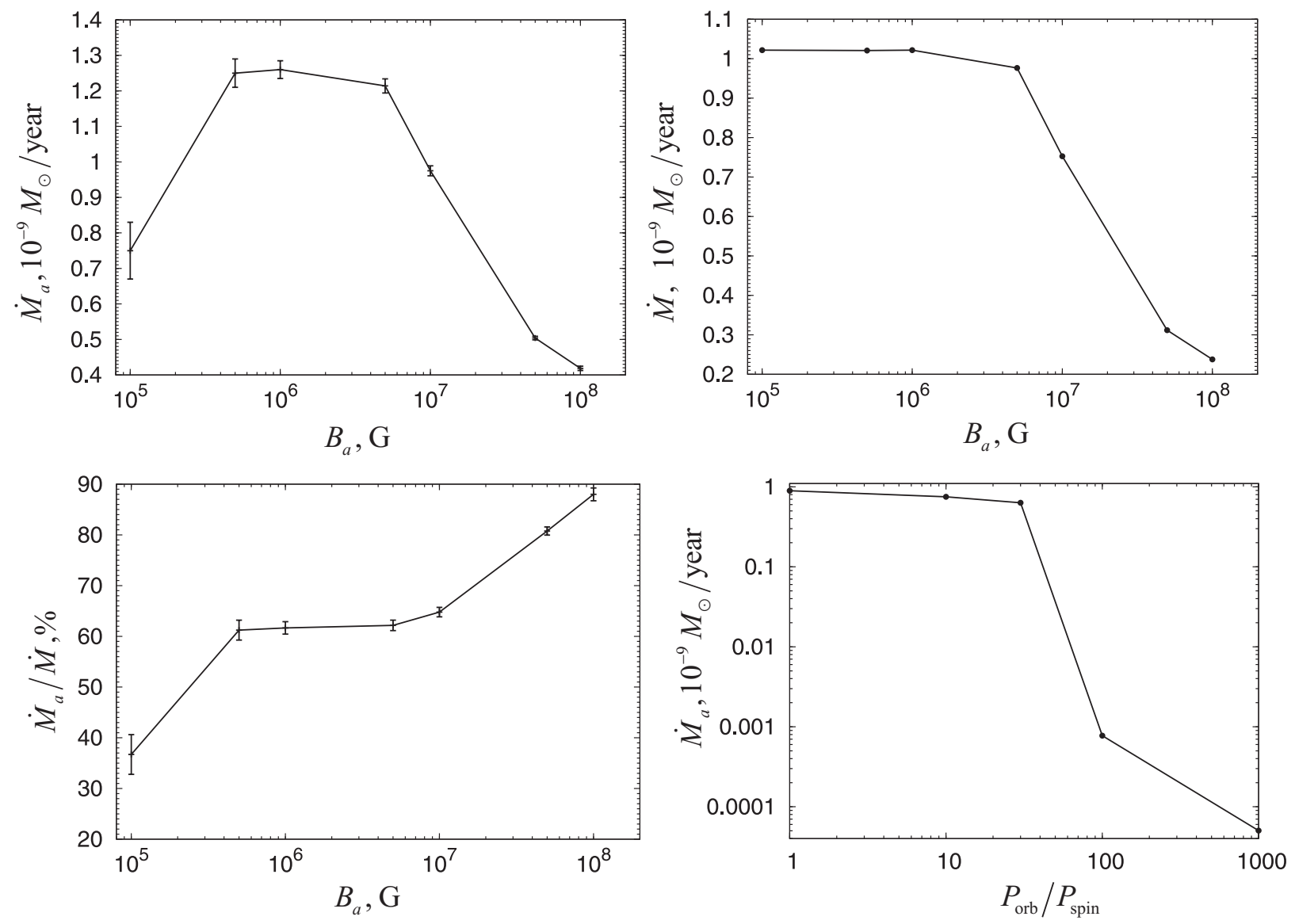

Figure 4: The dependence of the accretion rate $\dot{M}_{a}$ on value of the magnetic field $B_{a}$ (left row) in absolute values (top panel) and in \% from the mass transfer rate $\dot{M}$ (bottom panel). The vertical lines limit the specific amplitudes of the accretion rate variations. In the upper-right panel we show the mass transfer rate as a function of the value of magnetic field. In the lower-right panel we show the mass transfer rate as a function of the accretor's spin velocity in the model with $B_{a}=10^{5} \mathrm{G}$.

Analysis of the trajectories [11] shows that the stream comes very close to the accretor's surface at a distance $R_{\min }$. If $R_{\min }$ is larger than the radius of the magnetosphere $r_{m}$ the magnetic field does not strongly influence the motion of material. The stream rounds the star and intersects itself at a certain point. The further evolution of this flow results in the formation of an accretion disk in the system. If $R_{\min }$ is smaller than $r_{m}$ then the magnetic field starts to strongly influence the stream at a certain part of its trajectory. The action of electromagnetic forces in this region slows the stream down and causes loss of the angular momentum.

As a result the stream can not round the star and form an accretion disk. Thus, the boundary between the intermediate polars (with accretion disks) and polars (no accretion disks) is determined by the relation $r_{m}=R_{\min }$. If we calculate both the radii using the parameters of the SS Cygni we see that this relation is satisfied at $B_{a} \approx 10^{6} \mathrm{G}$ separating the two regimes of the flow. We should note that this estimate of magnetic field induction, separating the intermediate polars and polars, is more or less general for CVs, since the value of $q$ there varies insignificantly.

The typical flow structure in the polars is shown in Fig. 3, where, like in Figures 1 and 2, we plotted an iso-surface of density $\operatorname{logarithm} \lg \rho=-4.5$ (in units of $\left.\rho\left(L_{1}\right)\right)$ for the model $4\left(10^{7} \mathrm{G}\right)$. In the models $4-7$ no accretion disks form and material is accreted via funnel flows. In the models 4 and 5 the stream, starting at $L_{1}$ point, splits into two flows when approaching the accretor's surface. Then the first flows falls onto the northern magnetic pole of the accretor, the second onto the southern. With growing magnetic field the difference between the two poles becomes more obvious. In the model 6 , for example, only one accretion flow occurs that then ends up at the southern pole. In the model 7 the magnetic field is so strong that it almost entirely controls the flow structure in the Roche lobe of the accretor. The field captures material in the immediate vicinity of the inner Lagrangian point and, splitting 
it into two flows - more powerful southern and weaker northern, - transports it onto the accretor's surface along the field lines.

Let us now consider how the described variations of the flow structure influence the accretion rate. In the upper-left panel of Fig. 4 we plot the computed accretion rate $\dot{M}_{a}$ as a function of $B_{a}$. The vertical lines show specific values of the accretion rate variations. The main feature of this function is that it is non-monotonic. For the values $B_{a}<10^{6} G$ the accretion rate increases with increasing magnetic field and the amplitude of its variations decreases. At the point $B_{a}=10^{6} G$ the accretion rate approaches its maximal value. This can be explained by the fact that in the solutions with accretion disks the accretion rate is controlled by processes of angular momentum transfer. The growing magnetic field in intermediate polars makes magnetic breaking more effective and, hence, increases $\dot{M}_{a}$. After the point of maximum the accretion rate starts to decrease while $B_{a}$ continues to grow. As we have shown above starting at the point $B_{a}=10^{6} G$ no accretion disk forms in the system and the material is accreted via funnel flows. In this case the accretion rate is determined by the throughput of the stream. When the magnetic field grows the width of the stream decreases and the accretion rate falls.

Dependence of the accretion efficiency $\dot{M}_{a} / \dot{M}$ on the induction of magnetic field is shown in the lower-left panel of Fig. 4. With growing $B_{a}$ this value increases monotonically. In a transit range of magnetic field values (near $B_{a} \approx 10^{6} \mathrm{G}$ ) this dependence has an inflection point. The lowest effectiveness of accretion (30-40\%) is approached for intermediate polars with weak magnetic fields. This may be explained by the fact that their disks are large and a portion of material may leave the accretor's Roche lobe and form a common envelope of the binary system [12]. In the polars (with strong magnetic fields) almost all the material, flowing from the $L_{1}$ point onto the accretor, is accumulated and "enclosed" in the stream. Thus, in such systems the effectiveness of accretion reaches almost $100 \%$.

The non-monotonic shape of the $\dot{M}_{a}\left(B_{a}\right)$ curve, obtained in our calculations, does not contradict the monotonic character of the dependence of the accretion effectiveness on $B_{a}$. The point is that the mass transfer rate $\dot{M}$ also depends on the value of magnetic field. This dependence is shown in the upper-right diagram of Fig. 4. In Figure one can see that with growing $B_{a}$ the value of $\dot{M}$ monotonically decreases. In a range of weak fields ( $B_{a}<10^{6} \mathrm{G}$, intermediate polars) this decrease is almost invisible. However in strong fields $\left(B_{a}>10^{6} \mathrm{G}\right.$, polars) the value of the mass transfer rate sharply drops (by almost an order of magnitude). This behavior may be explained by the fact that the strong magnetic field of the accretor generates extra-pressure in the vicinity of the $L_{1}$ point, which prevents the outflow of the material from the donor's envelope. Thus, with growing $B_{a}$ the throughput of the accretion stream decreases.

\section{Influence of the Asynchronous Rotation of the White Dwarf on the Accretion Rate}

The influence of the accretor's spin-rotation on MHD flow structure in CVs can be characterized by a relation between the radius of the magnetosphere $r_{m}$ and the co-rotation radius $r_{c}$ that is a distance at which the velocity of rotation of the field lines is equal to the rotation velocity of material in the accretion disk. If the accretor rotates relatively slow $\left(r_{c}>r_{m}\right)$ then the rotation velocity of the field lines at the boundary of the magnetosphere is lower than the Keplerian. So the material, captured by the magnetic field can freely fall onto the accretor's surface. This regime may be called the "accretor" regime. When the accretor's rotation is fast enough $\left(r_{c}<r_{m}\right)$ a centrifugal barrier occurs at the boundary of the magnetosphere. This barrier prevents material from free falling onto the surface. This regime we may call the "propeller". In systems of AE Aqr type the rotation of the white dwarf is so fast that no accretion disk can form. Thus, for this regime we can use a term "super-propeller" [5].

To investigate how the asynchronous rotation of the accretor influences the flow structure in a close binary system we conducted four runs of 3D numerical simulations for the period relation of $P_{\text {spin }} / P_{\text {orb }}: 0.1$ ("accretor"); 0.033 ("equilibrium rotation"); 0.01 ("propeller"); and 0.001 ("super-propeller") [5]. To preserve the generality we again use the parameters of the SS Cygni. In all the runs the magnetic induction was set equal to $10^{5} \mathrm{G}$ and the magnetic axis is inclined by $\theta=30^{\circ}$.

In the models "accretor" and "equilibrium rotation" the flow structure is analogous to the model 1, considered in previous section. In the "propeller" regime, in the inner regions of the disk a magnetospheric cavity forms. Its radius is $0.05-0.1 A$. At the boundary of this cavity the Kelvin-Helmholtz instability develops. Since there is almost no material in the cavity, the accretion rate in the "propeller" model is close to zero. On the other hand the mass transfer continues and the mass of the disk grows. In the systems with an appropriate combination of parameters (disk mass, value of magnetic field and rotation velocity) the material at the boundary of the cavity at a certain moment can push the magnetosphere down and initiate a short period of accretion. After the exceptional mass has been removed the system returns into the "propeller" regime. In the "super-propeller" regime the material, issuing from the inner Lagrangian point, is captured by the 
rapidly rotating accretor's magnetosphere, acquires additional angular momentum and gets ejected out of the accretor's Roche lobe. The accretion rate in this case is almost zero.

In the lower-right panel of Fig. 4 we show dependence of the accretion rate on the parameter $P_{\text {orb }} / P_{\text {spin }}$ that characterizes the spin velocity of the accretor. The data shown are for the case of $B_{a}=10^{5} \mathrm{G}$. In Figure one can see that with the growing accretor's spin velocity the accretion rate decreases. If the white dwarf rotates relatively slow, which corresponds to the "accretor" regime, the accretion rate decrease is not significant in comparison with the case of synchronous rotation. However, when the spin velocity overcomes an equilibrium value the system enters the "ipropeller" regime and the accretion rate sharply drops. In fact, in the "propeller" and "super-propeller" regimes the accretion rate is determined by the accretion of material from the common envelope but not of the material, issuing from the inner Lagrangian point.

\section{Conclusions}

Using the results of 3D numerical MHD simulations, we have considered the influence of the magnetic field value $B_{a}$ and the spin-rotation velocity of the white dwarf on accretion processes in CVs. Analyzing the results of seven computational runs for models with $B_{a}$ equal to $10^{5} \mathrm{G}, 5 \times 10^{5} \mathrm{G}, 10^{6} \mathrm{G}, 5 \times 10^{6} \mathrm{G}, 10^{7} \mathrm{G}, 5 \times 10^{7} \mathrm{G}$ and $10^{8} \mathrm{G}$, we have found that the accretion rate $\dot{M}_{a}$ is a non-monotonic function of $B_{a}$. In the intermediate polars the growing $B_{a}$ makes the magnetic breaking more effective and, hence, increases $\dot{M}_{a}$. In the polars, where the accretion rate is determined by the throughput of the funnel flow the accretion rate decreases with growing $B_{a}$. The maximal value of the accretion rate occurs in the systems at a stage of transit between the intermediate polars and polars. This value is $\sim 60 \%$ of the initially set mass transfer rate.

We should note that unlike the absolute value of $\dot{M}_{a}$ the accretion efficiency $\dot{M}_{a} / \dot{M}$ monotonically increases with the increasing induction of magnetic field. In the polars it approaches the value of almost $100 \%$. This is because the mass transfer rate decreases with growing $B_{a}$, since strong magnetic field prevents the inflow of material into the accretor's Roche lobe through the inner Lagrangian point.

Analyzing the results of four computational runs of 3D MHD simulations with various values of the period ratio $\left(P_{\text {spin }} / P_{\text {orb }}=0.1,0.033,0.010 .001\right)$ and fixed $B_{a}=10^{5} \mathrm{G}$ we found that the accretion rate decreases with the increasing spin-rotation velocity. It is interesting to note that even in a system, living in the "propeller" regime, the average accretion rate may be not equal to zero if the combination of such parameters as the disk mass, value of magnetic field and rotation velocity can cause quasi-periodic accretion bursts (falling of the portion of the disk mass onto the accreting star).

\section{Acknowledgement}

This work was supported by the Basic Research Program of the Presidium of the Russian Academy of Sciences, the Russian Foundation for Basic Research Research (projects 11-02-00076, 12-02-00047, 13-02-00077, 13-02-00939), the Federal Targeted Program "Science and Science Education for Innovation in Russia 2009$2013 "$.

\section{References}

[1] Warner, B. : 1995, Cataclysmic variable stars, Cambridge University Press, Cambridge. doi:10.1017/СB09780511586491

[2] Campbell, C.G. : 1997, Magnetohydrodynamics in binary stars, Kluwer Acad., Dordrecht.

[3] Norton, A.J., , Wynn, J.A., Somerscales, R.V. : 2004, ApJ 614, 349. doi:10.1086/423333

[4] Zhilkin, A.G., Bisikalo, D.V. : 2010, Astron. Rep. $54,1063$.

[5] Zhilkin, A.G., Bisikalo, D.V., Boyarchuk, A.A. : 2012, Phys. Usp. 55, 115.

[6] Giovannelli, F., Gaudenzi, S., Rossi, C., Piccioni, A. P.J. : 1983, Acta Astronomica 33, 319.

[7] Zhilkin, A.G., Bisikalo, D.V. : 2009, Astron. Rep. 53, 436 .

[8] Zhilkin, A.G., Bisikalo, D.V. : 2010, Advances in Space Research 45, 437. doi:10.1016/j.asr.2009.09.006

[9] Boyarchuk, A.A., et al. : 2002, Mass transfer in close binary stars, Taylor \& Francis, London.

[10] Fridman, A.M., Bisikalo, D.V. : 2008, Phys. Usp. 51,551 .

[11] Lubow, S.H., Shu, F.H. : 1975, ApJ 198, 383. doi:10.1086/153614

[12] Sytov, A.Yu., et al. : 2007, Astron. Rep. 51, 836. doi:10.1134/S1063772907100083 


\section{DISCUSSION}

CHRISTIAN KNIGGE: You seemed to use SS Cyg as a prototype of DN intermediate polar, out what is the empirical evidence for this?

DMITRY BISIKALO: We just used SS Cyg as a DN illustrative example. The parameters of the SS Cyg (components masses, radii, separation, etc.) are quite typical for intermediate polars, so we can consider these numerical results as a good illustration of the flow structure in the intermediate polars.
GIORA SHAVIV: If I saw right, you do not have radiative transfer, only local energy sinks. How it works when the disk is optically thick?

DMITRY BISIKALO:Yes, this approach is strictly applicable for optically thin disks. If you consider optically thick disks it is necessary to add the radiative transfer in some form to the model, and we are working on this. Fortunately, we can use the assumption of optically thin disks for a significant sample of CVs. 